\title{
DIFFUSIONS WITH MEASUREMENT ERRORS. I. LOCAL ASYMPTOTIC NORMALITY
}

\author{
Arnaud GLOTER ${ }^{1}$ AND JEAN JACOD ${ }^{2}$
}

\begin{abstract}
We consider a diffusion process $X$ which is observed at times $i / n$ for $i=0,1, \ldots, n$, each observation being subject to a measurement error. All errors are independent and centered Gaussian with known variance $\rho_{n}$. There is an unknown parameter within the diffusion coefficient, to be estimated. In this first paper the case when $X$ is indeed a Gaussian martingale is examined: we can prove that the LAN property holds under quite weak smoothness assumptions, with an explicit limiting Fisher information. What is perhaps the most interesting is the rate at which this convergence takes place: it is $1 / \sqrt{n}$ (as when there is no measurement error) when $\rho_{n}$ goes fast enough to 0 , namely $n \rho_{n}$ is bounded. Otherwise, and provided the sequence $\rho_{n}$ itself is bounded, the rate is $\left(\rho_{n} / n\right)^{1 / 4}$. In particular if $\rho_{n}=\rho$ does not depend on $n$, we get a rate $n^{-1 / 4}$.
\end{abstract}

Mathematics Subject Classification. 60J60, 62F12, 62M05.

Received February 16, 2001. Revised October 24, 2001.

\section{INTRODUCTION}

1) In this paper as well as in the companion paper [6], we are interested in the following problem: let $X$ be a diffusion process on the time interval $[0,1]$, whose law depends on a parameter $\theta$. We observe this process at times $i / n$ for $i=0,1, \ldots, n$, and each observation is blurred by an error which is centered normal with variance $\rho_{n}$; in other words we observe $X_{\frac{i}{n}}+\sqrt{\rho_{n}} U_{i}$ where the $U_{i}$ 's are i.i.d. $\mathcal{N}(0,1)$, independent of the process. Our aim is to estimate the parameter $\theta$, knowing the noise level $\rho_{n}$.

This problem is clearly of pratical relevance but does not seem to have been studied so far, with the exception of a recent paper by Malyutov and Bayborodin [12] where no attempt towards optimality is made. The model is a hidden Markov model for which a lot is known (for "optimal" inference for such models, see e.g. Bickel and Ritov [1], [2], Jensen and Pedersen [9], Leroux [10] or Ryden [13]). However the situation at hand differs from ordinary Markov hidden models in which the hidden Markov chain is typically homogeneous ergodic, and time goes to infinity. Here the hidden Markov chain, i.e. the sequence $X_{i / n}, i=0,1, \ldots$, has a transition kernel depending on $n$ which degenerates as $n \rightarrow \infty$, while no ergodic property is relevant here.

\footnotetext{
Keywords and phrases: Statistics of diffusions, measurement errors, LAN property.

1 G.R.A.P.E., UMR 5113 du CNRS, Université Montesquieu (Bordeaux), Avenue Léon Duguit, 33608 Pessac, France; e-mail: gloter@montesquieu.u-bordeaux.fr

2 Laboratoire de Probabilités et Modèles Aléatoires, UMR 7599 du CNRS, Université Paris 6, 4 place Jussieu, 75252 Paris, France; e-mail: jj@ccr.jussieu.fr
} 
2) When there is no observation noise (i.e. $\rho_{n}=0$ ) the question is completely solved for diffusions of the form

$$
\mathrm{d} X_{t}=b_{t} \mathrm{~d} t+a\left(\theta, t, X_{t}\right) \mathrm{d} W_{t}, \quad \mathcal{L}\left(X_{0}\right)=\eta
$$

Here $W$ is a standard Brownian motion, and $\eta$ is an arbitrary initial law on $\mathbb{R}$, and $b$ is (non-anticipative) drift term which may depend on the path of $X$ or $W$. In fact, and of course assuming some smoothness of $a$, Donhal [3] proved the LAMN (= Local Asymptotic Mixed Normal) property in the 1-dimensional case for $a$ bounded away from 0 and $b_{t}=b\left(t, X_{t}\right)$, and in $[4,5]$ we have proved the LAMN property in the $d$-dimensional case when $a$ is a gradient and exhibited optimal estimators in all cases, and Gobet [7] has shown the LAMN property in general, when $a$ is non-degenerate. It turns out that the convergence rate in the LAMN property and for all optimal sequences of estimators is $1 / \sqrt{n}$.

Observe that in (1.1) the parameter $\theta$ does not appear in the drift $b_{t}$; it could appear but this would not improve the estimation since when $\theta$ appears in the drift only, it is not identifiable on the basis of even the full observation of $X$ over any bounded time interval.

3) When noise is present, several questions are natural. First, for a fixed noise level $\rho_{n}=\rho$ for all $n$, is it possible to have consistent estimators for $\theta$ and, if yes, at which rate? Second, if $\rho_{n}$ is small enough the noise is not going to affect the estimation procedures evoked above; what does exactly "small enough" mean? Third, what happens in between, when $\rho_{n}$ is small, but not "small enough"? Further, it is one thing to exhibit reasonable estimators, it is better to be able to study their asymptotic properties (such as their rates of convergence), it is even better to have "optimal" estimators and, for this, we need to describe the asymptotic behaviour of the relative likelihoods.

So an "optimal" program looks as follow: 1) study likelihoods (hopefully getting the LAMN property, as in the non-noisy case); 2) exhibit optimal sequences of estimators. We are unable so far to carry out this program completely. What we can do is as follows (under suitable assumptions, and with the notation $c(\theta, t, x)=$ $\left.a(\theta, t, x)^{2}\right)$ :

- Prove the LAMN (and in fact the LAN) property in the Gaussian martingale case, that is when $b_{t}(\omega)=0$ and $c(\theta, t, x)=c(\theta, t)$ does not depend on $x$ in (1.1). The corresponding Fisher information is $I(\theta)=$ $\int_{0}^{1} \iota(c(\theta, s), \dot{c}(\theta, s)) \mathrm{d} s$ for a suitable function $\iota$ on $\mathbb{R}^{2}(\dot{c}$ is the derivative of $\theta \rightsquigarrow c(\theta, s))$, and the rate $u_{n}$ depends only on the sequence $\rho_{n}$.

- In the case above we exhibit estimators $\widehat{\theta}_{n}$ which are asymptotically optimal, i.e. $\frac{1}{u_{n}}\left(\widehat{\theta}_{n}-\theta\right)$ converges to an $\mathcal{N}(0,1 / I(\theta))$ variable if the true value of the parameter is $\theta$.

- In the general case of (1.1), we can exhibit estimators $\widehat{\theta}_{n}$ such that $\frac{1}{u_{n}}\left(\widehat{\theta}_{n}-\theta\right)$ converges (with the same $u_{n}$ as above) to a mixed normal variable which, conditionally on the path of $X$, is $\mathcal{N}(0,1 / I(\theta))$, where $I(\theta)=\int_{0}^{1} \iota\left(c\left(\theta, s, X_{s}\right), \dot{c}\left(\theta, s, X_{s}\right)\right) \mathrm{d} s$ for the same function $\iota$ as above.

This first paper is devoted to the first problem above, while the second and third problems are studied in [6]. For simplicity we restrict ourselves to the 1-dimensional case for the parameter $\theta$ (not a real restriction), and also for the process $X$ : this could be relaxed at the cost of many more calculations.

The paper is organized as follows: in Section 2 we state the result and give a short description of the proof. In Section 3 we prove the result in the very simple case where $c(\theta, t)=c(\theta)$ depends on $\theta$ only. Section 4 is devoted to an auxiliary result which might be of independent interest and connects the LAN property for the initial experiments with the LAN property for "superexperiments" and "subexperiments". In Section 5 we give some technical results useful for this paper and/or [6]. Sections 6 and 7 are devoted to constructing the subexperiments and superexperiments respectively, and to proving the LAN property for both of them, while some results on matrices are gathered in an Appendix. 


\section{The RESUlT}

1) As said before, this paper is concerned with the following special case of (1.1); recall that $c=a^{2}$ :

$$
X_{t}=\int_{0}^{t} \sqrt{c(\theta, s)} \mathrm{d} W_{s}
$$

Here, $\Theta$ is any interval (bounded or not) of $\mathbb{R}$ and $c$ is a function: $\Theta \times[0,1] \mapsto \mathbb{R}_{+}$. The assumptions will be at least $\left(\mathrm{H} 1_{\theta}\right)$ and sometimes $\left(\mathrm{H} 2_{\theta}\right)$ or $\left(\mathrm{H} 3_{\theta}\right)$ below, for a given $\theta$ :

Hypothesis $\left(\mathbf{H} \mathbf{1}_{\theta}\right)$ : The function $\zeta \mapsto c(\zeta, x)$ is twice differentiable with partial first and second derivatives denoted by $\dot{c}$ and $\ddot{c}$, and $c, \dot{c}$ and $\ddot{c}$ are continuous on $\Theta \times[0,1]$. Further the function $c(\theta,$.$) does not vanish,$ and the function $\dot{c}(\theta,$.$) is not identically 0$.

Hypothesis $\left(\mathbf{H} \mathbf{2}_{\theta}\right)$ : The function $\dot{c}(\theta,$.$) does not vanish.$

Hypothesis ( $\left.\mathbf{H} 3_{\theta}\right)$ : The set $F=\{s \in[0,1]: \dot{c}(s, \theta)=0\}$ is the union of its connected components with positive length, plus a Borel set with Lebesgue measure equal to 0 . Moreover the function $\dot{c}(\theta,$.$) is Hölder-continuous$ with some index $\alpha \in(0,1]$.

Hypothesis $\left(\mathrm{H} 1_{\theta}\right)$ is a standard smoothness assumption, plus some non-degeneracy and identifiability at point $\theta$. $\left(\mathrm{H} 2_{\theta}\right)$ and $\left(\mathrm{H} 3_{\theta}\right)$ are additional identifiability assumptions at point $\theta$. Condition $\left(\mathrm{H} 3_{\theta}\right)$ rules out the case of a pathological set $F$, and the Hölder condition enables us to control $\dot{c}$ near $F$. ( $\left(\mathrm{H} 2_{\theta}\right)$ is indeed a strong assumption, while $\left(\mathrm{H} 3_{\theta}\right)$ is perhaps ugly-looking, but rather mild.

In this first paper we are interested in the LAN property but not in estimators, so we do not need a "global" identifiability assumption here.

Next we are given an i.i.d. sequence of $\mathcal{N}(0,1)$ variables $\left(U_{i}\right)$, independent of $W$. Our observations at stage $n$ consist in the finite sequence

$$
X_{i / n}+\sqrt{\rho_{n}} U_{i}, \quad i=0, \ldots, n,
$$

where $\rho_{n}$ is a known positive number for each $n$ (the observation for $i=0$ above gives no information about $\theta$, so one could as well take $i=1, \ldots, n$ in $(2.2))$.

At stage $n$ the simplest statistical experiment describing the previous scheme of observations consists in taking the state space to be $\mathbb{R}^{n+1}$ with the Borel $\sigma$-field, and for each $\theta$ the probability measure $P_{\theta}^{n}$ which is the law of the sequence in (2.2) when $X$ is given by (2.1). The measures $P_{\theta}^{n}$ are all equivalent, and we set $Z_{\zeta / \theta}^{n}=\mathrm{d} P_{\zeta}^{n} / \mathrm{d} P_{\theta}^{n}$ for the Radon-Nikodym derivative.

According to LeCam and Yang [11] for example, let us recall what the LAN property at point $\theta$, with rate $u_{n}$, is: this means that for any sequence $h_{n}$ of numbers going to a limit $h \in \mathbb{R}$ the sequence $Z_{\theta+u_{n} h_{n} / \theta}^{n}$ converges in law under $P_{\theta}^{n}$ to a limit of the form

$$
\exp \left(h U \sqrt{I(\theta)}-\frac{h^{2} I(\theta)}{2}\right), \quad \text { where } U \in \mathcal{N}(0,1) \quad \text { and } I(\theta)>0 .
$$

Then $I(\theta)$ is the (asymptotic) Fisher information.

The rate of convergence $u_{n}$ actually depends on the behaviour of the sequence $n \rho_{n}$. In fact, up to taking subsequences it is no real restriction to assume that this sequence converges in $[0, \infty]$ and, ruling out the totally 
uninteresting case where the sequence $\rho_{n}$ itself is unbounded, we see that three cases can occur:

$$
\left.\begin{array}{llll}
\text { Case } 1 & n \rho_{n} \rightarrow u=0: & \text { take } & u_{n}=1 / \sqrt{n} \\
\text { Case 2 } & n \rho_{n} \rightarrow u \in(0, \infty): & \text { take } & u_{n}=1 / \sqrt{n} \\
\text { Case } 3 & n \rho_{n} \rightarrow u=\infty, \sup _{n} \rho_{n}<\infty: & \text { take } & u_{n}=\left(\rho_{n} / n\right)^{1 / 4}
\end{array}\right\}
$$

Set also

$$
\iota_{u}(x, y)= \begin{cases}\frac{y^{2}}{2 x^{2}} & \text { if } u=0 \\ \frac{y^{2}(2+x / u)}{2 \sqrt{u} x^{3 / 2}(4+x / u)^{3 / 2}} & \text { if } 0<u<\infty \\ \frac{y^{2}}{8 x^{3 / 2}} & \text { if } u=\infty .\end{cases}
$$

The main result of this paper is then:

Theorem 2.1. Assume $\left(H 1_{\theta}\right)$. We have the LAN property at point $\theta$ and with the rates $u_{n}$ given above and the Fisher information

$$
I(\theta)=\int_{0}^{1} \iota_{u}(c(\theta, s), \dot{c}(\theta, s)) \mathrm{d} s
$$

in Cases 1 and 2 and also in Case 3 if further we have either $\left(H 2_{\theta}\right)$, or $\left(H 3_{\theta}\right)$ and the sequence $n^{1-4 \alpha} \rho_{n}$ is bounded (where $\alpha$ appears in $\left(\mathrm{H} 3_{\theta}\right)$ ).

The additional condition in Case 3 under $\left(\mathrm{H}_{\theta}\right)$ is of course automatically satisfied if $\alpha>1 / 4$. This theorem is also valid with slightly different observation schemes: namely if we observe the variables in $(2.2)$ for $i=1, \ldots, n$ only (perhaps a more natural setting), or if we observe the increments $X_{i / n}+\sqrt{\rho_{n}} U_{i}-\left(X_{(i-1) / n}+\sqrt{\rho_{n}} U_{i-1}\right)$ for $i=1, \ldots, n$.

Recall also the well known fact that, if there is no measurement error, the LAN property with rate $1 / \sqrt{n}$ and asymptotic Fisher information $\int_{0}^{1} \frac{\dot{c}(\rho, s)^{2}}{2 c(\theta, s)^{2}} \mathrm{~d} s$ holds; this is in accordance with the above: take $\rho_{n}=0$, so we are in Case 1.

2) Main steps of the proof. Although all involved variables are Gaussian, the problem is not simple, because of the complicated dependence structure of the observations.

Of course the case where $c(\theta, s)=c(\theta)$ does not depend on time is significantly easier, and we treat this case (referred to as the "homogeneous case") first, in Section 3 below: the method consists in making a linear transformation on the observed variables (2.2) to obtain independent variables; the key observation is that, due to the homogeneous structure, the orthogonal matrices which diagonalize the covariances are in fact independent of $\theta$ : then so is the linear transformation mentionned above, and the problem reduces to the classical situation of independent (non-identically distributed) centered Gaussian variables with unknown variances.

When $c$ depends on time, things are more complicated, and hinge upon two different ideas: the first idea is to split the sequence $1,2, \ldots, n$ into $l_{n}$ subsequences of length $k_{n}$, so that the variances of the increments $X_{i / n}-X_{(i-1) / n}$ are "almost" constant in $i$ within any given block of length $k_{n}$ when $\theta$ and $\zeta$ are close (an approximation is made here, but since $c$ is continuous in time this approximation is good if $k_{n} / n$ is small enough). Then each block can be treated as in the simple case above.

However, these blocks are not independent, so we need a second idea: we make the blocks independent by deleting the last observation in each of them: doing so we loose information, and get what one can call a 
"subexperiment". We can also make them independent by adding some observations, namely the values of $X_{i / n}$ and $U_{i}$ (instead of just $X_{i / n}+\sqrt{\rho_{n}} U_{i}$ ) at the end of each block: doing so increases information, and get what one can call a "superexperiment".

The difference in information between these experiments is so small that both the sub- and superexperiments have the LAN property with the same rate and the same asymptotic Fisher information: this yields (as shown in Sect. 4) that our original experiments, which are "in between" the sub- and superexperiments, share the same property.

It should be emphasized that even under $\left(\mathrm{H} 2_{\theta}\right)$, and even when $\left(\mathrm{H} 2_{\theta}\right)$ is strenghtened so that the function $\dot{c}$ is uniformly away from 0 , we still need to consider sub- and superexperiments.

3) Some general notation. Observe that there is no need to take $\mathbb{R}^{n+1}$ as our basic space. We stay closer to the structure of our processes by taking the state space $\Omega_{n}=\mathbb{R}^{2(n+1)}$, with the canonical variables $V_{0}, \ldots, V_{n}, U_{0}, \ldots, V_{n}$ and the Borel $\sigma$-field $\mathcal{H}_{n}$. Then $P_{\theta}^{n}$ is the unique probability measure under which the canonical variables are all independent, and the $U_{i}$ 's is $\mathcal{N}(0,1)$, and $V_{0}=0$ a.s., and the $V_{i}^{\prime} s$ for $i \geq 1$ are $\mathcal{N}\left(0, c_{i}^{n}(\theta)\right)$, with

$$
c_{i}^{n}(\theta)=\int_{(i-1) / n}^{i / n} c(\theta, s) \mathrm{d} s .
$$

That is, the variables in $(2.2)$ (for $i=0,1, \ldots, n$ ) have the same joint law than the variables $V_{0}+\ldots+V_{i}+\sqrt{\rho_{n}} U_{i}$ under $P_{\theta}^{n}$. The $\sigma$-field corresponding to the observations $(2.2)$ is

$$
\mathcal{F}_{n}=\sigma\left(V_{0}+\ldots+V_{i}+\sqrt{\rho_{n}} U_{i}: i=0, \ldots, n\right) .
$$

We also have $\mathcal{F}_{n}=\sigma\left(U_{0}, R_{1}, \ldots, R_{n}\right)$, where

$$
R_{i}=V_{i}+\sqrt{\rho_{n}}\left(U_{i}-U_{i-1}\right), \quad i=1, \ldots, n .
$$

\section{The homogeneous CASE}

In this section we wish to prove our result in the homogeneous case, because it minimizes the technicalities and clearly show why the rates are given by (2.4). In fact, since this is a particular example of the general case proved below, we feel free to slightly modify the observation scheme, in order to have even more simplicity: instead of observing the $\sigma$-field $\mathcal{F}_{n}$ of (2.8), we observe $\mathcal{F}_{n}^{\prime}=\mathcal{F}_{n} \bigvee \sigma\left(U_{n}\right)$ (or equivalently, the variables in (2.2) and also the variable $X_{1}$, or the variable $U_{n}$ : this will thus be a particular use of the "superexperiments" studied later).

So, in this section we suppose that $c(\zeta, s)=c(\zeta)$ for all $\zeta, s$. We fix $\theta$ and assume $\left(\mathrm{H} 1_{\theta}\right)$, which here implies $\left(\mathrm{H} 2_{\theta}\right)$, and in $(2.8)$ we have $c_{i}^{n}(\zeta)=c(\zeta) / n$. Our observation consists in the pair $\left(U_{0}, U_{n}\right)$ whose law in the same under all $P_{\zeta}^{n}$, and on the vector $S_{n}=\left(V_{1}+\sqrt{\rho_{n}} U_{1}, R_{2}, \ldots, R_{n-1}, V_{n}-\sqrt{\rho_{n}} U_{n-1}\right)$ which under $P_{\zeta}^{n}$ is independent of $\left(U_{0}, U_{n}\right)$ and is Gaussian centered with covariance matrix $C^{n}(\zeta)$ given by

$$
C^{n}(\zeta)_{i, j}= \begin{cases}\frac{c(\zeta)}{n}+\rho_{n} & \text { if } i=j=1 \text { or } i=j=n \\ \frac{c(\zeta)}{n}+2 \rho_{n} & \text { if } 2 \leq i=j \leq n-1 \\ -\rho_{n} & \text { if }|i-j|=1 \\ 0 & \text { otherwise. }\end{cases}
$$

This matrix can be diagonalized by an $n \times n$ orthogonal matrix $P^{n}$ which does not depend on $\zeta$, and its eigenvalues $\lambda_{u}^{n}(\zeta)$, increasingly ordered, can be explicitely computed: all this is proved in the Appendix, once 
observed that with the notation (8.4) we have $C^{n}(\zeta)=C\left(a_{n}(\zeta), 1, \rho_{n}\right)$ with $k=n$ and $b_{i}=1$ and $c_{i}=\frac{c(\theta)}{n}$ and $a_{n}(\zeta)=\frac{c(\zeta)-c(\theta)}{n}$. In particular, combining $(8.2)$ and Lemma 8.1, we get

$$
\lambda_{i}^{n}(\zeta)=\frac{c(\zeta)}{n}+2 \rho_{n}\left(1-\cos \left(\frac{(i-1) \pi}{n}\right)\right)
$$

Consider now the random vector $T^{n}=\left(T_{i}^{n}\right)_{1 \leq i \leq n}=P^{n \star} S^{n}$, and set $T_{i}^{\prime n}=T_{i}^{n} / \sqrt{\lambda_{i}^{n}(\theta)}$ : under $P_{\zeta}^{n}$, the components $T_{i}^{\prime n}$ are independent with laws $\mathcal{N}\left(0, \lambda_{i}^{n}(\zeta) / \lambda_{i}^{n}(\theta)\right)$ and independent of $\left(U_{0}, U_{n}\right)$, and in particular they are $\mathcal{N}(0,1)$ under $P_{\theta}^{n}$. Also, $\mathcal{F}_{n}^{\prime}$ is the $\sigma$-field generated by $U_{0}, U_{n}$, and $T_{i}^{\prime n}$ for $i=1, \ldots, n$. Then if $\left(h_{n}\right)$ is a sequence of reals going to a limit $h$, and if we set

$$
\delta_{i}^{n}=\frac{\lambda_{i}^{n}\left(\theta+u_{n} h_{n}\right)}{\lambda_{i}^{n}(\theta)}-1=\frac{c\left(\theta+u_{n} h_{n}\right)-c(\theta)}{c(\theta)+2 n \rho_{n}\left(1-\cos \left(\frac{(i-1) \pi}{n}\right)\right)},
$$

the likelihood $Z_{\theta+u_{n} h_{n} / \theta}^{n}=\left(\mathrm{d} P_{\theta+u_{n} h_{n}}^{n} / \mathrm{d} P_{\theta}^{n}\right)_{\mid \mathcal{F}_{n}^{\prime}}$ is given by:

$$
\log Z_{\theta+u_{n} h_{n} / \theta}^{n}=-\frac{1}{2} \sum_{i=1}^{n}\left(\log \left(1+\delta_{i}^{n}\right)-\left(T_{i}^{\prime n}\right)^{2} \frac{\delta_{i}^{n}}{1+\delta_{i}^{n}}\right)
$$

Since the $T_{i}^{\prime n}$ are i.i.d. $\mathcal{N}(0,1)$ under $P_{\theta}^{n}$, so in particular $\left(T_{i}^{\prime n}\right)^{2}$ has expectation 1 and variance 2 , a well known result (see e.g. Th. VIII-3.32 of [8]) shows that $Z_{\theta+u_{n} h_{n} / \theta}^{n}$ converges in law under $P_{\theta}^{n}$ to the variable defined in (2.3) (i.e., we have the desired LAN property) as soon as

$$
\sup _{1 \leq i \leq n}\left|\delta_{i}^{n}\right| \rightarrow 0, \quad \sum_{i=1}^{n}\left(\delta_{i}^{n}\right)^{2} \rightarrow 2 h^{2} I(\theta)
$$

where $I(\theta)$ is given by $(2.6)$, i.e. $I(\theta)=\iota_{u}(c(\theta), \dot{c}(\theta))$ here.

First, hypothesis $\left(\mathrm{H} 1_{\theta}\right)$ and (3.1) gives $\left|\delta_{i}^{n}\right| \leq C u_{n}$, hence giving the first part of (3.2). Second, $c\left(\theta+u_{n} h_{n}\right)-$ $c(\theta)=u_{n} h_{n}\left(\dot{c}(\theta)+O\left(u_{n}\right)\right)$, so with the notation (8.7) and using (3.1) again, we have

$$
\sum_{i=1}^{n}\left(\delta_{i}^{n}\right)^{2}=\left(\dot{c}(\theta)+O\left(u_{n}\right)\right)^{2} \frac{u_{n}^{2} h_{n}^{2}}{\pi n \rho_{n}^{2}} J_{2}^{\prime}\left(\frac{c(\theta)}{n \rho_{n}}, n\right) .
$$

Then (8.8) yields

$$
\frac{u_{n}^{2} h_{n}^{2}}{\pi n \rho_{n}^{2}} I_{2}\left(\frac{c(\theta)}{n \rho_{n}}\right) \leq \frac{u_{n}^{2} h_{n}^{2}}{\pi n \rho_{n}^{2}} J_{2}^{\prime}\left(\frac{c(\theta)}{n \rho_{n}}, n\right) \leq \frac{u_{n}^{2} h_{n}^{2}}{\pi n \rho_{n}^{2}} I_{2}\left(\frac{c(\theta)}{n \rho_{n}}\right)+\frac{u_{n}^{2} h_{n}^{2}}{c(\theta)^{2}}
$$

Since $u_{n} \rightarrow 0$, using (8.6) and studying separately the three cases in (2.4), we immediately deduce from $h_{n} \rightarrow h$ and (3.3) and (3.4) that the second part of (3.2) holds with $I(\theta)=\iota_{u}(c(\theta), \dot{c}(\theta))$.

\section{SUbeXPERIMENTS AND SUPEREXPERIMENTS}

The result of this section might be of independent interest. We have a sequence of statistical experiments $\left(\Omega_{n}, \mathcal{F}_{0}^{n}, \mathcal{F}_{1}^{n}, \mathcal{F}_{2}^{n},\left(P_{\theta}^{n}\right)\right)$, with increasing $\sigma$-fields $\mathcal{F}_{0}^{n} \subset \mathcal{F}_{1}^{n} \subset \mathcal{F}_{2}^{n}$. Let also $Z_{\zeta / \theta}^{n, i}$ be the $\mathcal{F}_{n}^{i}$-relative likelihood, i.e. $Z_{\zeta / \theta}^{n, i}=\left(\mathrm{d} P_{\zeta}^{n} / \mathrm{d} P_{\theta}^{n}\right)_{\mid \mathcal{F}_{i}^{n}}$. 
Theorem 4.1. Suppose that $Z_{\zeta_{n} / \theta}^{n, i}$ converges in law under $P_{\theta}^{n}$ to a limit $Y$ with $0<Y<\infty$ and $E(Y)=1$, for both $i=0$ and $i=2$ (the sequence of parameters $\zeta_{n}$ is completely arbitrary). Then the same convergence holds for $i=1$.

Proof. Set $Z_{i}^{n}=Z_{\zeta_{n} / \theta}^{n, i}$. Since $E_{\theta}^{n}\left(Z_{i}^{n}\right)=1$, the sequence $\left(Z_{0}^{n}, Z_{1}^{n}, Z_{2}^{n}\right)$ is uniformly tight. Up to taking subsequences, we may assume that $\left(Z_{0}^{n}, Z_{1}^{n}, Z_{2}^{n}\right)$ converges in law under $P_{\theta}^{n}$ to a limit denoted by $\left(Z_{0}, Z_{1}, Z_{2}\right)$. Our assumption yields $\mathcal{L}\left(Z_{0}\right)=\mathcal{L}\left(Z_{2}\right)=\mathcal{L}(Y)$.

Set $\mathcal{F}_{0}=\sigma\left(Z_{0}\right)$ and $\mathcal{F}_{1}=\sigma\left(Z_{0}, Z_{1}\right)$. Let $\psi_{p}$ be continuous functions on $\mathbb{R}$ with $0 \leq \psi_{p} \leq 1_{[-p, p]}$ and $\psi_{p}(x) \uparrow 1$. Finally let $\phi$ be a bounded continuous function on $\mathbb{R}^{2}$. For $i=0,1,2$ we have

$$
\begin{aligned}
& E_{\theta}^{n}\left(\phi\left(Z_{0}^{n}, Z_{1}^{n}\right) \psi_{p}\left(Z_{i}^{n}\right) Z_{i}^{n}\right) \rightarrow_{(n)} \quad E\left(\phi\left(Z_{0}, Z_{1}\right) \psi_{p}\left(Z_{i}\right) Z_{i}\right) \rightarrow_{(p)} \quad E\left(\phi\left(Z_{0}, Z_{1}\right) Z_{i}\right), \\
& E_{\theta}^{n}\left(\phi\left(Z_{0}^{n}, Z_{1}^{n}\right)\left(1-\psi_{p}\left(Z_{i}^{n}\right)\right) Z_{i}^{n}\right) \leq C E_{\theta}^{n}\left(Z_{i}^{n}-\psi_{p}\left(Z_{i}^{n}\right) Z_{i}^{n}\right)=C\left(1-E_{\theta}^{n}\left(\psi_{p}\left(Z_{i}^{n}\right) Z_{i}^{n}\right)\right) \\
& \rightarrow{ }_{(n)} C\left(1-E\left(\psi_{p}\left(Z_{i}\right) Z_{i}\right)\right) \rightarrow(p) \quad 0,
\end{aligned}
$$

hence $E_{\theta}^{n}\left(\phi\left(Z_{0}^{n}, Z_{1}^{n}\right) Z_{i}^{n}\right) \rightarrow E\left(\phi\left(Z_{0}, Z_{1}\right) Z_{i}\right)$. Now $E_{\theta}^{n}\left(\phi\left(Z_{0}^{n}, Z_{1}^{n}\right) Z_{1}^{n}\right)=E_{\theta}^{n}\left(\phi\left(Z_{0}^{n}, Z_{1}^{n}\right) Z_{2}^{n}\right)$ for each $n$, so $E\left(\phi\left(Z_{0}, Z_{1}\right) Z_{1}\right)=E\left(\phi\left(Z_{0}, Z_{1}\right) Z_{2}\right)$, which in turn yields $Z_{1}=E\left(Z_{2} \mid \mathcal{F}_{1}\right)$. One shows in a similar way that $Z_{0}=E\left(Z_{1} \mid \mathcal{F}_{0}\right)$.

Therefore $\sqrt{Z_{0}} \leq E\left(\sqrt{Z_{1}} \mid \mathcal{F}_{0}\right) \leq E\left(\sqrt{Z_{2}} \mid \mathcal{F}_{0}\right)$. Since $Z_{0}$ and $Z_{2}$ have the same law, $E\left(\sqrt{Z_{0}}\right)=E\left(\sqrt{Z_{2}}\right)$ and thus $Z_{0}=Z_{2}$ a.s. But $Z_{1}=E\left(Z_{2} \mid \mathcal{F}_{1}\right)$ a.s., hence $\mathcal{L}\left(Z_{1}\right)=\mathcal{L}(Y)$.

\section{Change of Probability}

Our aim is to prove Theorem 2.1, so we always assume $\left(\mathrm{H} 1_{\theta}\right)$ at least. That is, we fix $\theta$ and a sequence $h_{n}$ going to a limit $h \in \mathbb{R}$; if $Z_{n}$ is the relative likelihood $\mathrm{d} P_{\theta+u_{n} h_{n}}^{n} /\left.\mathrm{d} P_{\theta}^{n}\right|_{\mathcal{F}_{n}}$ (in restriction to $\mathcal{F}_{n}$ ), we need to prove that $Z_{n}$ converges in law under $P_{\theta}^{n}$ to $(2.3)$. By virtue of $\left(\mathrm{H} 1_{\theta}\right)$ there exists an $\varepsilon>0$ such that

$$
\frac{\varepsilon}{n} \leq c_{i}^{n}(\theta) \leq \frac{1}{n \varepsilon}, \quad \frac{\varepsilon}{n} \leq c_{i}^{n}\left(\theta+u_{n} h_{n}\right) \leq \frac{1}{n \varepsilon}, \quad n \geq 1,1 \leq i \leq n .
$$

As said before we wish to construct blocks such that within each of them one can consider $c_{i}^{n}(\theta)\left(\right.$ resp. $c_{i}^{n}(\theta+$ $\left.u_{n} h_{n}\right)$ ) in (2.7) as "almost" independent of $i$. For this, we first change $c_{i}^{n}\left(\theta+u_{n} h_{n}\right)$ in such a way that the asymptotic behaviour of the likelihood is not modified, and we will use the following well known result (see e.g. [4]): let $P^{\prime} n$ be an arbitrary probability measure on $\left(\Omega_{n}, \mathcal{H}_{n}\right)$ which is absolutely continuous w.r.t. $P_{\theta}^{n}$; let also $Z_{n}^{\prime}=\mathrm{d} P^{\prime n} /\left.\mathrm{d} P_{\theta}^{n}\right|_{\mathcal{F}_{n}}$ in restriction to $\mathcal{F}_{n}$.

Lemma 5.1. Assume that the variation distance (on $\left.\mathcal{H}_{n}\right)\left\|P^{\prime n}-P_{\theta+h_{n} u_{n}}^{n}\right\|_{v}$ goes to 0 . If the sequence $Z_{n}^{\prime}$ converges in law under $P_{\theta}^{n}$ to a limit $Y$ satisfying $0<Y<\infty$ and $E(Y)=1$, then the sequence $Z_{n}$ converges in law under $P_{\theta}^{n}$ to the same limit $Y$.

We will apply this lemma to the measure $P^{\prime n}$ under which the variables $U_{i}$ and $V_{i}$ are all independent, with $U_{i}$ being $\mathcal{N}(0,1)$ and $V_{0}=0$ a.s. and $V_{i}$ for $i=1, \ldots, n$ being $\mathcal{N}\left(0, c_{i}^{\prime n}\right)$, for numbers $c_{i}^{\prime n}>0$ to be constructed later.

Lemma 5.2. If

$$
n^{2} \sum_{i=1}^{n}\left|c_{i}^{\prime n}-c_{i}^{n}\left(\theta+u_{n} h_{n}\right)\right|^{2} \rightarrow 0
$$

then $\left\|P^{\prime n}-P_{\theta+u_{n} h_{n}}^{n}\right\|_{v} \rightarrow 0$. 
Proof. Let $\bar{Z}_{n}=\mathrm{d} P_{\theta+u_{n} h_{n}}^{n} / \mathrm{d} P^{\prime n}$ on the $\sigma$-field $\mathcal{H}_{n}$. Setting $c_{i}^{\prime \prime n}=c_{i}^{n}\left(\theta+u_{n} h_{n}\right)$, we have

$$
\bar{Z}_{n}=\prod_{i=1}^{n}\left(\sqrt{\frac{c_{i}^{\prime n}}{c_{i}^{\prime \prime n}}} \exp -\frac{V_{i}^{2}}{2}\left(\frac{1}{c_{i}^{\prime \prime n}}-\frac{1}{c_{i}^{\prime n}}\right)\right)
$$

Hence if $U$ is an $\mathcal{N}(0,1)$ variable, and since $E\left(\mathrm{e}^{a U^{2}}\right)=1 / \sqrt{1-2 a}$ for $a<1 / 2$, we get

$$
\begin{array}{r}
E^{\prime n}\left(\sqrt{\bar{Z}_{n}}\right)=\prod_{i=1}^{n}\left(\left(\frac{c_{i}^{\prime n}}{c_{i}^{\prime \prime n}}\right)^{1 / 4} E\left(\exp -\frac{U^{2}}{4}\left(\frac{c_{i}^{\prime n}}{c_{i}^{\prime \prime n}}-1\right)\right)\right) \\
=\prod_{i=1}^{n}\left(\left(\frac{c_{i}^{\prime n}}{c_{i}^{\prime \prime n}}\right)^{1 / 4}\left(1+\frac{1}{2}\left(\frac{c_{i}^{\prime n}}{c_{i}^{\prime \prime n}}-1\right)\right)^{-1 / 2}\right) \\
=\prod_{i=1}^{n}\left(\left(1+\delta_{i}^{n}\right)^{1 / 4}\left(1+\frac{1}{2} \delta_{i}^{n}\right)^{-1 / 2}\right),
\end{array}
$$

where $\delta_{i}^{n}=\frac{c_{i}^{\prime \prime n}-c_{i}^{\prime \prime \prime}}{c_{i}^{\prime \prime n}}$

The variation distance and the Hellinger distance induce the same topology (see e.g. [8], Chap. V), hence the result will be proved if we show that $E^{\prime n}\left(\left(1-\sqrt{\bar{Z}_{n}}\right)^{2}\right) \rightarrow 0$, which amounts to $E^{\prime n}\left(\sqrt{\bar{Z}_{n}}\right) \rightarrow 1$ (because $\left.E^{\prime n}\left(\bar{Z}_{n}\right)=1\right)$. This convergence holds as soon as $\sum_{i=1}^{n}\left(\delta_{i}^{n}\right)^{2} \rightarrow 0$, and this is implied by (5.2) because of (5.1).

Now we choose $c_{i}^{\prime n}$. Recall once more that $\theta$ and the sequence $h_{n}$ are fixed, while $u_{n}$ is given by (2.4). We set also

$$
c_{i}^{n}=c_{i}^{n}(\theta), \quad \dot{c}_{i}^{n}=\dot{c}_{i}^{n}(\theta) .
$$

Then we choose a subdivision $0=s_{1}^{n}<s_{2}^{n}<\ldots<s_{l_{n}+1}^{n}=1$ in such a way that

$$
\Delta_{n}:=\sup _{1 \leq m \leq l_{n}}\left(s_{m+1}^{n}-s_{m}^{n}\right) \rightarrow 0, \quad \inf _{1 \leq m \leq l_{n}}\left(s_{m+1}^{n}-s_{m}^{n}\right) \geq \frac{1}{n} .
$$

The choice of $l_{n}$ and of the $s_{m}^{n}$ 's will be made later. Then we set

$$
\begin{aligned}
& I_{n, m}=\left(s_{m}^{n}, s_{m+1}^{n}\right], \quad J_{n, m}=\left\{i: \frac{i}{n} \in I_{n, m}\right\}, \quad k_{n, m}=\# J_{n, m}, \quad i_{n, m}=\inf J_{n, m}, \\
& \left.\begin{array}{ll}
c_{\min }^{n, m}=\inf _{s \in I_{n, m}} c(\theta, s), & c_{\max }^{n, m}=\sup _{s \in I_{n, m}} c(\theta, s), \\
|\dot{c}|_{\min }^{n, m}=\inf _{s \in I_{n, m}}|\dot{c}(\theta, s)|, & |\dot{c}|_{\max }^{n, m}=\sup _{s \in I_{n, m}}|\dot{c}(\theta, s)|
\end{array}\right\} .
\end{aligned}
$$

Observe that $i \in J_{n, m} \Rightarrow\left|\dot{c}_{i}^{n}\right| \leq|\dot{c}|_{\max }^{n, m} / n$. We denote by $s_{n, m}$ any point achieving the maximum of $s \rightsquigarrow|\dot{c}(s, \theta)|$ on the closure of $I_{n, m}$, and we set $\dot{c}_{n, m}=\dot{c}\left(\theta, s_{n, m}\right) / n$, and $\ddot{c}_{n, m}=\ddot{c}_{i_{n, m}}^{n}(\theta)$, and

$$
i \in J_{n, m} \Rightarrow c_{i}^{\prime n}= \begin{cases}c_{i}^{n}+u_{n} h_{n} \dot{c}_{i}^{n}+\frac{u_{n}^{2} h_{n}^{2}}{2} \ddot{c}_{n, m} \frac{\dot{c}_{i}^{n}}{\dot{c}_{n, m}} & \text { if } \dot{c}_{n, m} \neq 0 \\ c_{i}^{n}+\frac{u_{n}^{2} h_{n}^{2}}{2} \ddot{c}_{n, m} & \text { if } \dot{c}_{n, m}=0 .\end{cases}
$$

Recall (5.1) and also that $\left|\dot{c}_{i}^{n}\right| \leq C / n$ and $\left|\ddot{c}_{n, m}\right| \leq C / n$ for some constant $C$ (below, $C$ denotes a constant which may change from line to line and depend on the function $c$, but not on $n$ and $i$ ). Hence, since $u_{n} h_{n} \rightarrow 0$, for all $n$ large enough $c_{i}^{\prime n}>0$ for all $i$. 
Observe that (5.6) is more complicated than just considering the function $c$ as constant on each interval $I(n, m)$. If we had chosen $c_{i}^{\prime n}$ independent of $i$ within each interval $I(n, m)$, then getting $(5.2)$ would necessitate restrictive conditions on the regularity of $\dot{c}$ and on the rate $\Delta_{n} \rightarrow 0$, implying in turn that the LAN property cannot be proved for all bounded sequence $\rho_{n}$ with this "simple" choice of $c_{i}^{\prime n}$. Our choice (5.6) is a modification of the Taylor expansion of $c_{i}^{n}\left(\theta+u_{n} h_{n}\right)$ chosen such that results of the Appendix apply to the covariance matrix of the observation under $P^{\prime n}$.

Lemma 5.3. The above-defined numbers $c_{i}^{\prime n}$ satisfy (5.2).

Proof. Taylor's formula yields for $i \in J_{n, m}$ :

$$
c_{i}^{n}\left(\theta+u_{n} h_{n}\right)-c_{i}^{\prime n}=\frac{u_{n}^{2} h_{n}^{2}}{2}\left(\ddot{c}_{i}^{n}\left(\theta_{i}^{n}\right)-\ddot{c}_{n, m}+\ddot{c}_{n, m}\left(1-\frac{\dot{c}_{i}^{n}}{\dot{c}_{n, m}}\right)\right),
$$

where $\theta_{i}^{n}$ is in between $\theta$ and $\theta+u_{n} h_{n}$, and with the convention $0 / 0=1$ (recall that if $\dot{c}_{n, m}=0$ we also have $\left.\dot{c}_{i}^{n}(\theta)=0\right)$. In Cases 1 or 2 , we have $\left|c_{i}^{n}\left(\theta+u_{n} h_{n}\right)-c_{i}^{\prime n}\right| \leq C n^{-2}$ and (5.2) follows.

Case 3 needs more attention. By $\left(\mathrm{H} 1_{\theta}\right)$ and (5.4) there is a sequence $\varepsilon_{n} \rightarrow 0$ having

$$
s_{m}^{n} \leq s \leq t \leq s_{m+1}^{n},|\zeta-\theta| \leq u_{n}\left|h_{n}\right| \Rightarrow|\ddot{c}(\zeta, t)-\ddot{c}(\theta, s)|+|\dot{c}(\theta, t)-\dot{c}(\theta, s)| \leq \varepsilon_{n} .
$$

Then

$$
i \in J_{n, m} \quad \Rightarrow \quad\left|\ddot{c}_{i}^{n}\left(\theta_{i}^{n}\right)-\ddot{c}_{n, m}\right|+\left|\dot{c}_{i}^{n}-\dot{c}_{n, m}\right| \leq \frac{\varepsilon_{n}}{n}
$$

Since $\left|\ddot{c}_{n, m}\right| \leq C / n$ and $\left|\dot{c}_{i}^{n}\right| \leq\left|\dot{c}_{n, m}\right|$ and $u_{n}^{4} \leq C / n$, we deduce that

$$
n^{2}\left|c_{i}^{n}\left(\theta+u_{n} h_{n}\right)-c_{i}^{\prime n}\right|^{2} \leq \begin{cases}\frac{C}{n} \varepsilon_{n}^{2} & \text { if } \dot{c}_{n, m}=0 \\ \frac{C}{n} & \text { if } 0<\left|\dot{c}_{n, m}\right|<\sqrt{\varepsilon_{n}} / n \\ \frac{C}{n} \varepsilon_{n} & \text { if }\left|\dot{c}_{n, m}\right| \geq \sqrt{\varepsilon_{n}} / n .\end{cases}
$$

Hence, in order to obtain (5.2) it suffices to prove that $\frac{1}{n} \sum_{m \in K_{n}} k_{n, m} \rightarrow 0$, where $K_{n}=\left\{m: 0<\left|\dot{c}_{n, m}\right|<\right.$ $\left.\sqrt{\varepsilon_{n}} / n\right\}$. This is immediate under $\left(\mathrm{H} 2{ }_{\theta}\right)$.

Now we complement the notation of $\left(\mathrm{H} 3_{\theta}\right)$. The open set $F^{c}$ is the finite or countable union of its connected components $\left(a_{j}, b_{j}\right)$, the number of which being denoted by $M \leq \infty$. The closed set $F$ is the finite or countable union of its connected components $\left[a_{j}^{\prime}, b_{j}^{\prime}\right]$ (with $a_{j}^{\prime}<b_{j}^{\prime}$, the number of which is denoted by $M^{\prime} \leq \infty$ ), plus possibly a set $F^{\prime}$ having $\lambda\left(F^{\prime}\right)=0$, where $\lambda$ is the Lebesgue measure.

Observe that by (5.4) we have $k_{n, m} \leq 2 n \lambda\left(I_{n, m}\right)$. For all $i$ and $\eta>0$, for all $n$ large enough we have $|\dot{c}(\theta, s)| \geq \sqrt{\varepsilon_{n}}$ when $s \in\left[a_{i}+\eta, b_{i}-\eta\right]$ (recall that $\varepsilon_{n} \rightarrow 0$ ); hence for all $n$ large enough $\left|\dot{c}_{n, m}\right| \geq \sqrt{\varepsilon_{n}} / n$ for any interval $\left.I_{n, m} \subset\right] a_{i}+\eta, b_{i}-\eta\left[\right.$. We also have $\dot{c}(s, \theta)=0$ if $s \in\left[a_{i}^{\prime}, b_{i}^{\prime}\right]$, hence $\dot{c}_{n, m}=0$ as soon as $I_{n, m} \subset\left[a_{i}^{\prime}, b_{i}^{\prime}\right]$. Therefore, since each interval $I_{n, m}$ has length smaller than $\Delta_{n}$, we get for any integer $N$, any $\eta>0$ and any $n$ bigger than some $n_{N, \eta}$ :

$$
\frac{1}{n} \sum_{m \in K_{n}} k_{n, m} \leq 2\left(1-\sum_{i=1}^{N \wedge M}\left(b_{i}-a_{i}-2 \Delta_{n}-2 \eta\right)-\sum_{i=1}^{N \wedge M^{\prime}}\left(b_{i}^{\prime}-a_{i}^{\prime}-2 \Delta_{n}\right)\right) .
$$

Since $\Delta_{n} \rightarrow 0$ we obtain

$$
\limsup _{n} \frac{1}{n} \sum_{m \in K_{n}} k_{n, m} \leq 2\left(1-\sum_{i=1}^{N \wedge M}\left(b_{i}-a_{i}-2 \eta\right)-\sum_{i=1}^{N \wedge M^{\prime}}\left(b_{i}^{\prime}-a_{i}^{\prime}\right)\right) .
$$


The union of all $\left(a_{j}, b_{j}\right)$ and $\left[a_{i}^{\prime}, b_{i}^{\prime}\right]$ being $F^{\prime c}$, and $\lambda\left(F^{\prime c}\right)=1$, and $\eta>0$ is arbitrary: henceforth $\frac{1}{n} \sum_{m \in K_{n}}$ $k_{n, m} \rightarrow 0$.

Let us end this section by describing how to gather all previous results for proving Theorem 2.1. We first fix $\theta$. Then for each $n$ we construct two $\sigma$-fields $\mathcal{F}_{n}^{-}$and $\mathcal{F}_{n}^{+}$with $\mathcal{F}_{n}^{-} \subset \mathcal{F}_{n}^{+} \subset \mathcal{H}_{n}$ (corresponding to the sub- and superexperiment) and subdivisions $\left(s_{m}^{n,-}\right)$ and $\left(s_{m}^{n,+}\right)$ satisfying (5.4); then with each sequence $h_{n}$ converging to a limit $h$ we associate $c_{i}^{\prime n-}$ (resp. $c_{i}^{\prime n+}$ ) by (5.6) and the corresponding measures $P^{\prime n-}$ (resp. $P^{\prime n+}$ ) described above, and finally the relative likelihoods $Z_{n}^{-}=\mathrm{d} P^{\prime n-} /\left.\mathrm{d} P_{\theta}^{n}\right|_{\mathcal{F}_{n}^{-}}\left(\right.$resp. $\left.Z_{n}^{+}=\mathrm{d} P^{\prime n+} /\left.\mathrm{d} P_{\theta}^{n}\right|_{\mathcal{F}_{n}^{+}}\right)$.

Putting together Theorem 4.1 and Lemmas 5.1, 5.2 and 5.3, we readily obtain the following corollary:

Corollary 5.4. Suppose that both $Z_{n}^{-}$and $Z_{n}^{+}$converge in law under $P_{\theta}^{n}$ to the same limit given by (2.3) with $I(\theta)$ given by (2.6), for any choice of sequences $h_{n}$ converging to $h$. Then the experiments $\left(\Omega_{n}, \mathcal{F}_{n},\left(P_{\zeta}^{n}\right)\right)$ have the $L A N$ property at point $\theta$ with Fisher information $I(\theta)$, for any choice of $\sigma$-fields $\mathcal{F}_{n}$ such that $\mathcal{F}_{n}^{-} \subset \mathcal{F}_{n} \subset \mathcal{F}_{n}^{+}$.

Therefore for proving Theorem 2.1 it is enough to exhibit the $\sigma$-fields $\mathcal{F}_{n}^{-}$and $\mathcal{F}_{n}^{+}$with $\mathcal{F}_{n}^{-} \subset \mathcal{F}_{n} \subset \mathcal{F}_{n}^{+}$for every observed $\sigma$-field $\mathcal{F}_{n}$ of interest (and in particular the one given by (2.8)), and such that the convergence assumptions of the previous corollary hold true.

\section{LAN PROPERTY FOR SUBEXPERIMENTS}

First we need to construct the subexperiments and the subdivisions satisfying (5.4). We choose $\gamma \in\left(\frac{1}{2}, 1\right)$ and set

The following is obvious:

$$
k_{n}=\left[n^{\gamma}\right], \quad l_{n}=\left[\frac{n}{k_{n}}\right] .
$$

$$
k_{n} \rightarrow \infty, \quad l_{n} \rightarrow \infty, \quad u_{n}^{2} l_{n} \rightarrow 0
$$

Then we set $s_{m}^{n}=m k_{n} / n$ if $0 \leq m \leq l_{n}$ and $s_{l_{n}+1}^{n}=1$. Clearly (5.4) holds, and we use all notation of Section 5 , in particular $c_{i}^{\prime n}$ given by (5.6) and $P^{\prime n}$ as defined before Lemma 5.2. Observe that $k_{n, m}=k_{n}$ if $1 \leq m \leq l_{n}-1$.

Next, denote by $M_{n}$ the set of all indices $m$ in $\left\{1, \ldots, l_{n}-1\right\}$ such that $s \rightsquigarrow \dot{c}(\theta, s)$ does not vanish on the interval $I_{n, m}$. Then set (recall $(2.9)$ for $R_{i}$ ):

$$
\mathcal{F}_{n}^{-}=\sigma\left(R_{i}: k_{n}(m-1)+1 \leq i \leq k_{n} m-1, m \in M_{n}\right)
$$

Our aim is to prove the following, where $Z_{n}^{-}=\mathrm{d} P^{\prime n} /\left.\mathrm{d} P_{\theta}^{n}\right|_{\mathcal{F}_{n}^{-}}$:

Proposition 6.1. Under $\left(H 1_{\theta}\right)$, and with the previous notation, the sequence $Z_{n}^{-}$converges in law under $P_{\theta}^{n}$ to the limit described in (2.3), with $u_{n}$ given by (2.4) and $I(\theta)$ given by (2.6).

Proof. 1) The observations corresponding to the $\sigma$-field $\mathcal{F}_{n}^{-}$are naturally divided into \# $M_{n}$ blocks: if $m \in M_{n}$, let $R^{n, m}$ denote the column vector whose components are $R_{i}^{n, m}=R_{(m-1) k_{n}+i}$ for $i=1, \ldots, k_{n}-1$. By construction, the vectors $R^{n, m}$ for $m \in M_{n}$ are centered Gaussian and independent under both $P_{\theta}^{n}$ and $P^{\prime n}$.

Let us fix $m \in M_{n}$. The covariance matrix of $R^{n, m}$ under $P_{\theta}^{n}\left(\right.$ resp. $\left.P^{\prime n}\right)$ is denoted by $C^{n, m}\left(\right.$ resp. $\left.C^{\prime n, m}\right)$ : observe that all $\dot{c}_{i}^{n}$ for $i \in J_{n, m}$ have the same sign, say $\alpha_{n}(=+1$ or $=-1)$; so with the notation (8.4) we have $C^{n, m}=C\left(0,2, \rho_{n}\right)$ and $C^{\prime n, m}=C\left(v_{n, m}, 2, \rho_{n}\right)$, provided $k=k_{n, m}-1$ and $c_{i}=c_{(m-1) k_{n}+i}^{n}$ and $b_{i}=b_{i}^{n, m}=\left|\dot{c}_{(m-1) k_{n}+i}^{n}\right|$ and $v_{n, m}=\alpha_{n} u_{n} h_{n}\left(1+\frac{u_{n} h_{n} \ddot{c}_{n, m}}{2 \dot{c}_{n, m}}\right)$ (recall (5.6)).

Next, consider the vectors $S^{n, m}$ with components $S_{i}^{n, m}=R_{i}^{n, m} / \sqrt{b_{i}^{n, m}}$ : under $P_{\theta}^{n}$ (resp. $P^{\prime n}$ ) it is centered Gaussian with covariance $\widehat{C}^{n, m}$ (resp. $\widehat{C}^{\prime n, m}$ ) associated with $C^{n, m}$ and $C^{\prime n, m}$ as in Lemma 8.1. Write $\widehat{\lambda}_{i}^{n, m}$ and $\hat{\lambda}_{i}^{\prime n, m}, i=1, \ldots, k_{n}-1$, for the increasingly ordered eigenvalues of these matrices. From Lemma 8.1 we 
know that the orthogonal matrix which diagonalizes $\widehat{C}^{n, m}$ and $\widehat{C}^{\prime n, m}$ is the same, say $P^{n, m}$, and also that (use notation (8.2)):

$$
\left.\begin{array}{c}
\widehat{\lambda}_{i}^{\prime n, m}=\widehat{\lambda}_{i}^{n, m}+v_{n, m}, \\
\frac{c_{\min }^{n, m}}{|\dot{c}|_{\max }^{n, m}}\left(1+\frac{n \lambda_{i}\left(2, \rho_{n}\right)}{c_{\max }^{n, m}}\right) \leq \hat{\lambda}_{i}^{n, m} \leq \frac{c_{\max }^{n, m}}{|\dot{c}|_{\min }^{n, m}}\left(1+\frac{n \lambda_{i}\left(2, \rho_{n}\right)}{c_{\min }^{n, m}}\right)
\end{array}\right\} .
$$

Hence the vector $Y^{n, m}=\left(P^{n, m}\right)^{\star} S^{n, m}$ has components $Y_{i}^{n, m}$ which are independent normal centered with variances $\widehat{\lambda}_{i}^{n, m}$ and $\widehat{\lambda}_{i}^{\prime n, m}$ under $P_{\theta}^{n}$ and $P^{\prime n}$ respectively. Then the variables $Y_{i}^{\prime n, m}=Y_{i}^{n, m} / \sqrt{\widehat{\lambda}_{i}^{n, m}}$ are i.i.d. $\mathcal{N}(0,1)$ under $P_{\theta}^{n}$, and independent $\mathcal{N}\left(0, \hat{\lambda}_{i}^{\prime n, m} / \widehat{\lambda}_{i}^{n, m}\right)$ under $P^{\prime n}$ (when $m$ and $i$ vary); furthermore these variables generate the $\sigma$-field $\mathcal{F}_{n}^{-}$: so exactly as in Section 3 , and if we set

$$
\delta_{i}^{n, m}=\frac{\widehat{\lambda}_{i}^{\prime n, m}-\widehat{\lambda}_{i}^{n, m}}{\widehat{\lambda}_{i}^{n, m}}=\frac{v_{n, m}}{\widehat{\lambda}_{i}^{n, m}},
$$

we have

$$
\log Z_{n}^{-}=-\frac{1}{2} \sum_{m \in M_{n}} \sum_{i=1}^{k_{n, m}-1}\left(\log \left(1+\delta_{i}^{n, m}\right)-\left(Y_{i}^{\prime n, m}\right)^{2} \frac{\delta_{i}^{n, m}}{1+\delta_{i}^{n, m}}\right) .
$$

Therefore, as in Section 3 again, for obtaining the desired claim it is enough to prove the following two conditions:

$$
\begin{gathered}
\sup _{1 \leq i \leq k_{n, m}-1, m \in M_{n}}\left|\delta_{i}^{n, m}\right| \rightarrow 0, \\
F_{n}:=\sum_{m \in M_{n}} \widehat{F}_{n, m} \rightarrow 2 I(\theta) h^{2}, \quad \text { where } \widehat{F}_{n, m}=\sum_{i=1}^{k_{n, m}-1}\left|\delta_{i}^{n, m}\right|^{2} .
\end{gathered}
$$

2) First, $\left|v_{n, m}\right| \leq C u_{n}\left(1+u_{n} /|\dot{c}|_{\min }^{n, m}\right)$, and (6.3) and $\left(\mathrm{H} 1_{\theta}\right)$ yield $0<1 / \widehat{\lambda}_{i}^{n, m} \leq C|\dot{c}|_{\max }^{n, m} \leq C$. Hence (6.4) yields $\left|\delta_{i}^{n, m}\right| \leq C u_{n}$, and we have (6.6).

Next, with the notation $W_{n}=\sup _{1 \leq m \leq l_{n}}\left(c_{\max }^{n, m} / c_{\min }^{n, m}\right)^{2}$ and $\phi(x, a)=a+2(1-\cos x)$ we have by (8.2):

$$
\frac{v_{n, m}^{2}\left(|\dot{c}|_{\min }^{n, m}\right)^{2}}{n^{2} \rho_{n}^{2} W_{n} \phi\left(\frac{i \pi}{k_{n, m}}, \frac{c_{\min }^{n, m}}{n \rho_{n}}\right)^{2}} \leq\left|\delta_{i}^{n, m}\right|^{2} \leq \frac{v_{n, m}^{2}\left(|\dot{c}|_{\max }^{n, m}\right)^{2} W_{n}}{n^{2} \rho_{n}^{2} \phi\left(\frac{i \pi}{k_{n, m}}, \frac{c_{\max }^{n, m}}{n \rho_{n}}\right)^{2}} .
$$

Taking into account the value of $v_{n, m}$, hypothesis $\left(\mathrm{H}_{\theta}\right)$ and the fact that $|\dot{c}|_{\min }^{n, m} \leq|\dot{c}|_{\max }^{n, m}=n\left|\dot{c}_{n, m}\right|$, we deduce that $\left|v_{n, m}^{2}-u_{n}^{2} h_{n}^{2}\right| \leq C u_{n}^{2} h_{n}^{2}\left(\frac{u_{n}}{|\dot{c}|_{\text {max }}^{n, m}}+\frac{u_{n,}^{2}}{\left(|\vec{c}|_{\max }^{n, m}\right)^{2}}\right)$, hence

$$
\begin{aligned}
& \frac{u_{n}^{2} h_{n}^{2}}{n^{2} \rho_{n}^{2} W_{n} \phi\left(\frac{i \pi}{k_{n, m}}, \frac{c_{\min }^{n, m}}{n \rho_{n}}\right)^{2}}\left(\left(|\dot{c}|_{\min }^{n, m}\right)^{2}-C u_{n}|\dot{c}|_{\min }^{n, m}-C u_{n}^{2}\right) \\
& \leq\left|\delta_{i}^{n, m}\right|^{2} \leq \frac{u_{n}^{2} h_{n}^{2} W_{n}}{n^{2} \rho_{n}^{2} \phi\left(\frac{i \pi}{k_{n, m}}, \frac{c_{\max }^{n, m}}{n \rho_{n}}\right)^{2}}\left(\left(|\dot{c}|_{\max }^{n, m}\right)^{2}+C u_{n}|\dot{c}|_{\max }^{n, m}+C u_{n}^{2}\right)
\end{aligned}
$$


3) Now we use the notation $I_{2}$ and $J_{2}$ of (8.6) and (8.7). From (6.7) and (6.8) we deduce

$$
\begin{aligned}
& \frac{u_{n}^{2} h_{n}^{2} k_{n, m}}{n^{2} \rho_{n}^{2} W_{n} \pi} J_{2}\left(\frac{c_{\min }^{n, m}}{n \rho_{n}}, k_{n, m}\right)\left(\left(|\dot{c}|_{\min }^{n, m}\right)^{2}-C u_{n}|\dot{c}|_{\min }^{n, m}-C u_{n}^{2}\right)^{+} \\
& \quad \leq \widehat{F}_{n, m} \leq \frac{u_{n}^{2} h_{n}^{2} k_{n, m} W_{n}}{n^{2} \rho_{n}^{2} \pi} J_{2}\left(\frac{c_{\max }^{n, m}}{n \rho_{n}}, k_{n, m}\right)\left(\left(|\dot{c}|_{\max }^{n, m}\right)^{2}+C u_{n}|\dot{c}|_{\max }^{n, m}+C u_{n}^{2}\right)
\end{aligned}
$$

Thus if we set $w_{n}(y)=\frac{u_{n}^{2}}{n \rho_{n}^{2} \pi} I_{2}\left(y / n \rho_{n}\right)$, we deduce from $(8.8)$ and $\left(\mathrm{H} 1_{\theta}\right)$ that

$$
\begin{aligned}
& \frac{h_{n}^{2}}{W_{n}}\left(\frac{k_{n, m}}{n} w_{n}\left(c_{\min }^{n, m}\right)-C u_{n}^{2}\right)^{+}\left(\left(|\dot{c}|_{\min }^{n, m}\right)^{2}-C u_{n}|\dot{c}|_{\min }^{n, m}-C u_{n}^{2}\right)^{+} \\
& \leq \widehat{F}_{n, m} \leq h_{n}^{2} W_{n} \frac{k_{n, m}}{n} w_{n}\left(c_{\max }^{n, m}\right)\left(\left(|\dot{c}|_{\max }^{n, m}\right)^{2}+C u_{n}|\dot{c}|_{\max }^{n, m}+C u_{n}^{2}\right) .
\end{aligned}
$$

Notice that if we set $\widehat{F}_{n, m}=0$ for $m \in\left\{1, \ldots, l_{n}-1\right\} \backslash M_{n}$, and since then we also have $|\dot{c}|_{\text {min }}^{n, m}=0$, the estimates (6.10) are also valid in this case. Furthermore, with this convention, we have $F_{n}=\sum_{m=1}^{l_{n}-1} \widehat{F}_{n, m}$.

4) Using (8.6), one checks that the sequence $w_{n}$ converges to the following function $w$, uniformly on the interval $[\varepsilon, 1 / \varepsilon]$ :

$$
w(y)= \begin{cases}\frac{1}{y^{2}} & \text { in Case } 1 \\ \frac{2+y / u}{\sqrt{u} y^{3 / 2}(4+y / u)^{3 / 2}} & \text { in Case } 2 \\ \frac{1}{4 y^{3 / 2}} & \text { in Case } 3 .\end{cases}
$$

Let $r_{m}^{n}$ and $r_{m}^{\prime n}$ be points in the closed interval $\bar{I}_{n, m}$ such that $c\left(\theta, r_{m}^{n}\right)=c_{\max }^{n, m}$ and $c\left(\theta, r_{m}^{\prime n}\right)=c_{\min }^{n, m}$. By $\left(\mathrm{H} 1_{\theta}\right)$ there exists $\zeta_{n} \rightarrow 0$ such that $u_{n} \leq \zeta_{n}$ and

$$
\left.\begin{array}{l}
1-\zeta_{n} \leq \frac{1}{W_{n}} \leq W_{n} \leq 1+\zeta_{n}, \quad y \in\left[\varepsilon, \frac{1}{\varepsilon}\right] \Rightarrow\left|w_{n}(y)-w(y)\right| \leq \zeta_{n}, \\
\left.|| \dot{c}\left(\theta, r_{m}^{n}\right)|-| \dot{c}\right|_{\max } ^{m, n}\left|\leq \zeta_{n}, \quad\right|\left|\dot{c}\left(\theta, r_{m}^{\prime n}\right)\right|-|\dot{c}|_{\min }^{m, n} \mid \leq \zeta_{n}
\end{array}\right\}
$$

Hence by $(6.10)$ and $\varepsilon \leq c(\theta, s) \leq \frac{1}{\varepsilon}$ and the fact that $w_{n}(y)$ for $y \in[\varepsilon, 1 / \varepsilon]$ and $|\dot{c}|_{\max }^{n, m}$ and $u_{n}$ are uniformly bounded, and also because $k_{n, m}=k_{n}$ for $m \leq l_{n}-1$ :

$$
\begin{aligned}
& \frac{\left(1-\zeta_{n}\right) h_{n}^{2}}{l_{n}-1} \sum_{m=1}^{l_{n}-1} \frac{\left(l_{n}-1\right) k_{n}}{n} w\left(c\left(\theta, r_{m}^{\prime n}\right)\right) \dot{c}\left(\theta, r_{m}^{\prime n}\right)^{2}-C \zeta_{n}-C u_{n}^{2} l_{n} \\
& \leq F_{n} \leq \frac{\left(1+\zeta_{n}\right) h_{n}^{2}}{l_{n}-1} \sum_{m=1}^{l_{n}-1} \frac{\left(l_{n}-1\right) k_{n}}{n} w\left(c\left(\theta, r_{m}^{n}\right)\right) \dot{c}\left(\theta, r_{m}^{n}\right)^{2}+C \zeta_{n} .
\end{aligned}
$$

Observe that $\frac{\left(l_{n}-1\right) k_{n}}{n} \rightarrow 1$, while $u_{n}^{2} l_{n} \rightarrow 0$ by (6.1). Since the sums in (6.13) are Riemann sums, using the uniform convergence of $w_{n}$ to $w$ on $[\varepsilon, 1 / \varepsilon]$ and recalling (5.1), we deduce (6.7) and our proof is finished. 


\section{LAN PROPERTY FOR SUPEREXPERIMENTS}

First we need to construct the superexperiments and the subdivisions satisfying (5.4). Here again we choose $\gamma \in\left(\frac{1}{2}, 1\right)$ and set

$$
k_{n}=\left[n^{\gamma}\right], \quad l_{n}^{\prime}=\left[\frac{n}{k_{n}}\right], \quad k_{n}^{\prime}=n-\left(l_{n}^{\prime}-1\right) k_{n} .
$$

Set $t_{m}^{n}=k_{n} m / n$ if $0 \leq m \leq l_{n}^{\prime}-1$, and $t_{l_{n}^{\prime}}^{n}=1$, and $I_{n, m}^{\prime}=\left(t_{m-1}^{n}, t_{m}^{n}\right]$ for $1 \leq m \leq l_{n}^{\prime}$. Set also $J_{n, m}^{\prime}=\{i$ : $\left.i / n \in I_{n, m}^{\prime}\right\}$.

Denote by $K_{n}^{\prime}$ the set of all indices $m$ such that either $s \rightsquigarrow \dot{c}(\theta, s)$ does not vanish on $I_{n, m}^{\prime}$, or $\dot{c}(\theta, s)=0$ for all $s \in I_{n, m}^{\prime}$, and $K_{n}^{\prime \prime}=\left\{1, \ldots, l_{n}^{\prime}\right\} \backslash K_{n}^{\prime}$. If $m \in K_{n}^{\prime \prime}$, we divide $J_{n, m}^{\prime}$ into sub-intervals of integers which are of maximal length and such that either $\dot{c}_{i}^{n}>0$ or $\dot{c}_{i}^{n}<0$ for all $i$ in any of these sub-intervals, plus all the "intervals" $\{i\}$ such that $\dot{c}_{i}^{n}=0$. We have thus divided $\{1, \ldots, n\}$ into $l_{n}$ intervals (the $J_{n, m}^{\prime}$ 's for $m \in K_{n}^{\prime}$, and the sub-intervals of the $J_{n, m}^{\prime}$ 's for $\left.m \in K_{n}^{\prime \prime}\right)$ : these intervals, ordered according to the natural order, are denoted $J_{n, m}$, for $m=1, \ldots, l_{n}$. Let $k_{n, m}=\# J_{n, m}$, and call $i_{n, m}$ and $j_{n, m}$ the smallest and the biggest points in $J_{n, m}$, and by convention $i_{n, 0}=j_{n, 0}=0$ : we have $i_{n, m} \leq j_{n, m}$ and $i_{n, 1}=1$ and $i_{n, m+1}=j_{n, m}+1$ and $j_{n, l_{n}}=n$. It remains to set $s_{m}^{n}=\frac{j_{n, m-1}}{n}$ for $m=1, \ldots, l_{n}$, and $I_{n, m}=\left(s_{m}^{n}, s_{m+1}^{n}\right]$ : we have (5.4) and (5.5). Observe also that under $\left(\mathrm{H} 2_{\theta}\right)$ we have $K_{n}^{\prime}=\left\{1, \ldots, l_{n}^{\prime}\right\}$ and $l_{n}=l_{n}^{\prime}$ and $I_{n, m}=I_{n, m}^{\prime}$.

Finally, we set

$$
\mathcal{F}_{n}^{+}=\mathcal{F}_{n} \bigvee \sigma\left(U_{j_{n, m}}: m=0, \ldots, l_{n}\right)
$$

Our aim is to prove the following, where $Z_{n}^{+}=\mathrm{d} P^{\prime n} /\left.\mathrm{d} P_{\theta}^{n}\right|_{\mathcal{F}_{n}^{+}}$:

Proposition 7.1. Assume $\left(H 1_{\theta}\right)$ and, in Case 3, either $\left(H 2_{\theta}\right)$ or $\left(H 3_{\theta}\right)$ plus the boundedness of the sequence $\rho_{n} n^{1-4 \alpha}$. Then with the previous notation, the sequence $Z_{n}^{+}$converges in law under $P_{\theta}^{n}$ to the limit described in (2.3), with $u_{n}$ given by (2.4) and $I(\theta)$ given by (2.6).

Then Propositions 6.1 and 7.1, together with Corollary 5.4, will end the proof of Theorem 2.1, once noticed that $\mathcal{F}_{n}^{-} \subset \mathcal{F}_{n} \subset \mathcal{F}_{n}^{+}$.

Proof. 1) Set

$$
R_{i}^{\prime}= \begin{cases}V_{i}+\sqrt{\rho_{n}} U_{i} & \text { if } i=i_{n, m}<j_{n, m} \text { for some } m \\ V_{i}-\sqrt{\rho_{n}} U_{i-1} & \text { if } i=j_{n, m}>i_{n, m} \text { for some } m \\ V_{i} & \text { if } i=i_{n, m}=j_{n, m} \text { for some } m \\ R_{i} & \text { otherwise. }\end{cases}
$$

Then, comparing with (2.8) and (7.2), we obtain

$$
\mathcal{F}_{n}^{+}=\sigma\left(U_{j_{n, m}}: m=0, \ldots, l_{n} ; R_{i}^{\prime}, i=1, \ldots, n\right)
$$

Denote by $K_{n}$ the set of indices $m$ such that $J_{n, m}$ is one of the initial sets $J_{n, m^{\prime}}^{\prime}$ on which $\dot{c}(\theta,$.$) does not vanish,$ and by $H_{n}$ the set of indices $m$ such that $J_{n, m}$ is one of the initial sets $J_{n, m^{\prime}}^{\prime}$ on which $\dot{c}(\theta,$.$) is identically 0$, and $L_{n}$ the set of indices $m$ such that $i_{n, m}=j_{n, m}$, and finally $M_{n}=\left\{1, \ldots, l_{n}\right\} \backslash\left(L_{n} \cup K_{n} \cup H_{n}\right)$. Under both 
$P_{\theta}^{n}$ and $P^{\prime n}$, the observations are divided into independent blocks, namely

$\left.\begin{array}{l}\text { 1. the } U_{j_{n, m}} \text { for } m=0, \ldots, l_{n} \text { (they are } \mathcal{N}(0,1) \text { under both probabilities), } \\ \text { 2. the } R^{\prime n, m}=V_{i_{n, m}} \text { for } m \in L_{n} \text {, } \\ \text { 3. the column vectors } R^{\prime n, m} \text { whose components are } R_{i}^{\prime n, m}=R_{i_{n, m}+i-1}^{\prime} \text { for } \\ i=1, \ldots, k_{n, m} \text { for } m \in K_{n} \cup H_{n} \cup M_{n}\end{array}\right\}$.

Note that under $\left(\mathrm{H} 2_{\theta}\right)$ we have $H_{n}=L_{n}=M_{n}=\emptyset$.

2) In order to study $R_{n, m}^{\prime}$, we essentielly repeat what was done for Proposition 6.1, with some slight modifications.

First suppose that $m \in K_{n} \cup H_{n} \cup M_{n}$. Under $P_{\theta}^{n}$ (resp. $P^{\prime n}$ ) the vector $R^{\prime n, m}$ is centered Gaussian with covariance matrix $C^{n, m}$ (resp. $C^{\prime n, m}$ ). If $m \in K_{n} \cup M_{n}$ (resp. $m \in H_{n}$ ) the $\dot{c}_{i}^{n}$ for $i \in J_{n, m}$ all have the same sign, say $\alpha_{n}(=+1$ or $=-1)\left(\right.$ resp. $\dot{c}_{i}^{n}=0$ for $\left.i \in J_{n, m}\right)$, hence $C^{n, m}=C\left(0,1, \rho_{n}\right)(c f$. (8.4)) and $C^{\prime n, m}=C\left(v_{n, m}, 1, \rho_{n}\right)$, provided $k=k_{n, m}$ and $c_{i}=c_{i_{n, m}+i-1}^{n}$ and $b_{i}=b_{i}^{n, m}=\left|\dot{c}_{i_{n, m}+i-1}^{n}\right|$ and $v_{n, m}=$ $\alpha_{n} u_{n} h_{n}\left(1+\frac{u_{n} h_{n} \ddot{c}_{n, m}}{2 \ddot{c}_{n, m}}\right)\left(\right.$ resp. $\left.v_{n, m}=u_{n}^{2} h_{n}^{2} \ddot{c}_{n, m} / 2\right)$.

Now, for $m \in K_{n} \cup M_{n}$, we introduce the vector $S^{n, m}$ with components $S_{i}^{n, m}=R_{i}^{\prime n, m} / \sqrt{b_{i}^{n, m}}$ : on the one side the $\sigma$-fields generated by $R^{\prime n, m}$ and by $S^{n, m}$ coincide. On the other side these vectors are centered Gaussian under both $P_{\theta}^{n}$ and $P^{\prime n}$, and their covariance matrices are the matrices $\widehat{C}^{n, m}$ and $\widehat{C}^{\prime n, m}$ associated with $C^{n, m}$ and $C^{\prime n, m}$ as in Lemma 8.1. Write $\widehat{\lambda}_{i}^{n, m}$ and $\widehat{\lambda}_{i}^{\prime n, m}, i=1, \ldots, k_{n, m}$ for the increasingly ordered eigenvalues of these matrices. From Lemma 8.1 the orthogonal matrix which diagonalizes $\widehat{C}^{n, m}$ and $\widehat{C}^{\prime n, m}$ is the same, say $P^{n, m}$. Hence the vector $Y^{n, m}=\left(P^{n, m}\right)^{\star} S^{n, m}$ has components $Y_{i}^{n, m}$ which are independent normal centered with variances $\widehat{\lambda}_{i}^{n, m}$ and $\widehat{\lambda}_{i}^{\prime n, m}$ under $P_{\theta}^{n}$ and $P^{\prime n}$ respectively. Hence the variables $Y_{i}^{\prime n, m}=Y_{i}^{n, m} / \sqrt{\widehat{\lambda}_{i}^{n, m}}$ are independent, $\mathcal{N}(0,1)$ under $P_{\theta}^{n}$ and $\mathcal{N}\left(0, \widehat{\lambda}_{i}^{\prime n, m} / \widehat{\lambda}_{i}^{n, m}\right)$ under $P^{\prime n}$, and they generate the same $\sigma$-field than $R^{\prime n, m}$. Further, we have (6.3) with $\lambda_{i}\left(1, \rho_{n}\right)$ instead of $\lambda_{i}\left(2, \rho_{n}\right)$, and we define $\delta_{i}^{n, m}$ by (6.4).

On the other hand, if $m \in L_{n}$ the 1-dimensional variable $Y_{i_{n, m}}^{\prime n, m}=R^{\prime n, m} / \sqrt{c_{i_{n, m}}^{n}}$ is $\mathcal{N}(0,1)$ (resp. $\mathcal{N}\left(0, c_{i_{n, m}}^{\prime n} /\right.$ $\left.\left.c_{i_{n, m}}^{n}\right)\right)$ under $P_{\theta}^{n}\left(\right.$ resp. $\left.P^{\prime n}\right)$; in this case we set $\delta_{i_{n, m}}^{n, m}=\left(c_{i_{n, m}}^{\prime n}-c_{i_{n, m}}^{n}\right) / c_{i_{n, m}}^{n}$.

Therefore, using the independence of all variables occuring in (7.3), we obtain as in Section 3 that

$$
\log Z_{n}^{+}=-\frac{1}{2} \sum_{m=1}^{l_{n}} \sum_{i=1}^{k_{n, m}}\left(\log \left(1+\delta_{i}^{n, m}\right)-\left(Y_{i}^{\prime n, m}\right)^{2} \frac{\delta_{i}^{n, m}}{1+\delta_{i}^{n, m}}\right),
$$

and here again it remains to prove the analogues of (6.6) and (6.7), except that $M_{n}$ is replaced by $\left\{1,2, \ldots, l_{n}\right\}$ and that we must take $\widehat{F}_{n, m}=\sum_{i=1}^{k_{n, m}}\left|\delta_{i}^{n, m}\right|^{2}$. Again as in Proposition 6.1 we have $\left|\delta_{i}^{n, m}\right| \leq C u_{n}$, hence (6.6) holds.

If $m \in K_{n} \cup H_{n} \cup M_{n}$, and in view of (8.2), we get the estimate (6.8) except that we should replace $i \pi / k_{n, m}$ by $(i-1) \pi / k_{n, m}$. Therefore (6.9) holds with $J_{2}^{\prime}$ instead of $J_{2}$, and by (8.8) we have the following estimate, analogous to (6.10) (with the same notation $W_{n}, w_{n}$ as in Proposition 6.1; of course if $m \in H_{n}$ we have $\dot{c}_{\min }^{n, m}=\dot{c}_{\max }^{n, m}=0$ )

$$
\begin{aligned}
& \frac{h_{n}^{2} k_{n, m}}{W_{n} n} w_{n}\left(c_{\min }^{n, m}\right)\left(\left(|\dot{c}|_{\min }^{n, m}\right)^{2}-C u_{n}|\dot{c}|_{\min }^{n, m}-C u_{n}^{2}\right)^{+} \leq \widehat{F}_{n, m} \\
& \leq h_{n}^{2} W_{n}\left(\frac{k_{n, m}}{n} w_{n}\left(c_{\max }^{n, m}\right)+C u_{n}^{2}\right)\left(\left(|\dot{c}|_{\max }^{n, m}\right)^{2}+C u_{n}|\dot{c}|_{\max }^{n, m}+C u_{n}^{2}\right)
\end{aligned}
$$


In particular since $W_{n}$ and $|\dot{c}|_{\max }^{n, m}$ and $w_{n}(y)$ for $y \in[\varepsilon, 1 / \varepsilon]$ are uniformly bounded, we get

$$
m \in K_{n} \cup H_{n} \cup M_{n} \Rightarrow \widehat{F}_{n, m} \leq C \frac{k_{n, m}}{n} .
$$

4) Suppose that $\left(\mathrm{H} 2_{\theta}\right)$ holds. Then $l_{n}=l_{n}^{\prime}$ and $H_{n}=L_{n}=M_{n}=\emptyset$, and $k_{n, m}=k_{n}$ for all $m \leq l_{n}-1$. Then using (6.10) and with $\zeta_{n}$ as in Proposition 6.1, we obtain (compare to (6.13); we use the same notation for $r_{m}^{n}$ and $r_{m}^{\prime n}$ and $w$; the last extra term on the right below comes from $(7.1,7.5)$ and the fact that $\left.k_{n, l_{n}}=k_{n}^{\prime} \leq 2 k_{n}\right)$ :

$$
\begin{aligned}
& \frac{\left(1-\zeta_{n}\right) h_{n}^{2}}{l_{n}^{\prime}-1} \sum_{m=1}^{l_{n}^{\prime}-1} \frac{\left(l_{n}^{\prime}-1\right) k_{n}}{n} w\left(c\left(\theta, r_{m}^{\prime n}\right)\right) \dot{c}\left(\theta, r_{m}^{\prime n}\right)^{2}-C \zeta_{n} \leq F_{n} \\
& \leq \frac{\left(1+\zeta_{n}\right) h_{n}^{2}}{l_{n}^{\prime}-1} \sum_{m=1}^{l_{n}^{\prime}-1} \frac{\left(l_{n}^{\prime}-1\right) k_{n}}{n} w\left(c\left(\theta, r_{m}^{n}\right)\right) \dot{c}\left(\theta, r_{m}^{n}\right)^{2}+C \zeta_{n}+C u_{n}^{2} l_{n}^{\prime}+\frac{C}{n^{1-\gamma}}
\end{aligned}
$$

Then we conclude exactly as in Proposition 6.1 that (6.7) holds and our result is proved.

5) It remains to examine the situation when $\left(\mathrm{H} 2_{\theta}\right)$ fails. Let $\eta(r)=\sup (|\dot{c}(\theta, t)-\dot{c}(\theta, s)|: s, t \in[0,1],|s-t| \leq r)$. Then $\eta(r)$ decreases to 0 when $r \downarrow 0$, and under $\left(\mathrm{H} 3_{\theta}\right)$ we even have $\eta(r) \leq C r^{\alpha}$.

When $m \in L_{n}$, we have $|\dot{c}|_{\max }^{n, m} \leq C(1 / n)^{\alpha}$ because $\dot{c}(\theta,$.$) vanishes at a distance less than 1 / n$ from the point $i_{n, m} / n$. Hence, in view of (5.6), $\widehat{F}_{n, m} \leq C\left(u_{n}^{2}(1 / n)^{2 \alpha}+u_{n}^{4}\right)$. This and (7.5) yield (since $k_{n, m}=1$ when $\left.m \in L_{n}\right)$ :

$$
\sum_{m \in L_{n} \cup M_{n}} \widehat{F}_{n, m} \leq C \beta_{n}\left(1+u_{n}^{2} n\left(\frac{1}{n}\right)^{2 \alpha}+n u_{n}^{4}\right), \quad \text { where } \quad \beta_{n}=\frac{1}{n} \sum_{m \in L_{n} \cup M_{n}} k_{n, m} .
$$

Note that $\beta_{n}$ is the Lebesgue measure of the union of all $I_{n, m}^{\prime}\left(\right.$ for $\left.m=1, \ldots, l_{n}^{\prime}\right)$ on which $\dot{c}(\theta,$.$) vanishes but$ is not identically 0 , and for such an $m$ we have $0<\sup _{s \in I_{n, m}^{\prime}}|\dot{c}(\theta, s)| \leq \sqrt{\varepsilon_{n}}$, where $\varepsilon_{n}=C\left(1 / n^{1-\gamma}\right)^{2 \alpha}$. Then we prove exactly as in Lemma 5.3 that $\beta_{n} \rightarrow 0$. The sequence $n u_{n}^{4}$ is always bounded; the sequence $u_{n}^{2} n^{1-2 \alpha}$ tends to 0 in Cases 1 and 2 and is bounded in Case 3 as soon as $\rho_{n} n^{1-4 \alpha}$ stays bounded, an assumption which is made in Theorem 2.1 under $\left(\mathrm{H} 3_{\theta}\right)$. Therefore we deduce that

$$
\gamma_{n}:=\sum_{m \in L_{n} \cup M_{n}} \widehat{F}_{n, m} \rightarrow 0 .
$$

Now we combine (6.10) for $m \in K_{n} \cup H_{n}$ (then $m$ corresponds to one of the $l_{n}$ original sets $\left.I_{n, m^{\prime}}^{\prime}\right)$ with (7.7), to obtain estimates similar to (7.6): an upper bound for $F_{n}$ is clearly

$$
\frac{\left(1+\zeta_{n}\right) h_{n}^{2}}{l_{n}^{\prime}-1} \sum_{m=1}^{l_{n}^{\prime}-1} \frac{\left(l_{n}^{\prime}-1\right) k_{n}}{n} w\left(c\left(\theta, r_{m}^{n}\right)\right) \dot{c}\left(\theta, r_{m}^{n}\right)^{2}+C \zeta_{n}+C u_{n}^{2} l_{n}^{\prime}+\frac{C}{n^{1-\gamma}}+\gamma_{n}
$$

A lower bound for $F_{n}$ is like in (7.6) except that the sum is taken over all $m \leq l_{n}^{\prime}-1$ which belong to $K_{n}^{\prime}$ (that is, such that the original interval $I_{n, m}^{\prime}$ equals one of the $I_{n, j}$ for $\left.j \in K_{n} \cup H_{n}\right)$. But since when $m \notin K_{n}^{\prime}$ we have $\left|\dot{c}\left(\theta, r_{m}^{n}\right)\right| \leq C n^{(1-\gamma) \alpha}$, a lower bound for $F_{n}$ is also given by

$$
\frac{\left(1-\zeta_{n}\right) h_{n}^{2}}{l_{n}^{\prime}-1} \sum_{m=1}^{l_{n}^{\prime}-1} \frac{\left(l_{n}-1\right) k_{n}}{n} w\left(c\left(\theta, r_{m}^{\prime n}\right)\right) \dot{c}\left(\theta, r_{m}^{\prime n}\right)^{2}-C \zeta_{n}-C \beta_{n} n^{(1-\gamma) \alpha}
$$

Then, once more like in Proposition 6.1, we conclude from (7.7) and (7.8) and (7.9) that (6.7) holds, and we are finished. 


\section{Appendix: Some Results on matrices}

Here we give some elementary results on tridiagonal matrices which come naturally as covariance matrices of our observations.

Let $\rho$ be a positive number and $\beta$ be either 1 or 2 . We introduce the following $k \times k$ nonnegative symmetric and tridiagonal matrix $D(\beta, \rho)$ :

$$
D(\beta, \rho)_{i, j}= \begin{cases}\beta \rho & \text { if } i=j=1 \text { or } i=j=k \\ 2 \rho & \text { if } 2 \leq i=j \leq k-1 \\ -\rho & \text { if }|i-j|=1 \\ 0 & \text { otherwise. }\end{cases}
$$

The eigenvalues $\left(\lambda_{i}(\beta, \rho): i=1, \ldots, k\right)$ of $D(\beta, \rho)$, increasingly ordered, can be explicitely computed

$$
\lambda_{i}(2, \rho)=2 \rho\left(1-\cos \left(\frac{i \pi}{k+1}\right)\right), \quad \lambda_{i}(1, \rho)=2 \rho\left(1-\cos \left(\frac{(i-1) \pi}{k}\right)\right) .
$$

Next, let $b_{1}, \ldots, b_{k}$ and $c_{1}, \ldots, c_{k}$ be positive numbers, and set

$$
\left.\begin{array}{ll}
b_{\min }=\inf _{i} b_{i}, & b_{\max }=\sup _{i} b_{i} \\
c_{\min }=\inf _{i} c_{i}, & c_{\max }=\sup _{i} c_{i}
\end{array}\right\}
$$

Let also $a \in \mathbb{R}$ be such that $c_{i}+a b_{i}>0$ for all $i$. With all these we associate the following $k \times k$ nonnegative symmetric and tridiagonal matrix $C(a, \beta, \rho)=C\left(\left(b_{i}\right),\left(c_{i}\right), a, \beta, \rho\right)$ :

$$
C(a, \beta, \rho)_{i, j}= \begin{cases}c_{i}+a b_{i}+\beta \rho & \text { if } i=j=1 \text { or } i=j=k \\ c_{i}+a b_{i}+2 \rho & \text { if } 2 \leq i=j \leq k-1 \\ -\rho & \text { if }|i-j|=1 \\ 0 & \text { otherwise. }\end{cases}
$$

Finally let $\Delta$ be the matrice diagonal matrix with entries $\Delta_{i, i}=b_{i}^{-1 / 2}$, and the two (symmetric nonnegative) matrices $\widehat{C}=\Delta C(0, \beta, \rho) \Delta$ and $\widehat{C}^{\prime}=\Delta C(a, \beta, \rho) \Delta$. We denote by $\widehat{\lambda}_{i}$ and $\widehat{\lambda}_{i}^{\prime}$ the eigenvalues of $\widehat{C}$ and $\widehat{C}^{\prime}$, increasingly ordered, and by $\Lambda$ and $\Lambda^{\prime}$ the associated diagonal matrices, and by $P$ and $P^{\prime}$ orthogonal matrices such that $\widehat{C}=P \Lambda P^{*}$ and $\widehat{C}^{\prime}=P^{\prime} \Lambda^{\prime} P^{\prime *}$.

Lemma 8.1. With the above notation, we have

(i) $\widehat{\lambda}_{i}^{\prime}=\widehat{\lambda}_{i}+a$;

(ii) $\frac{c_{\min }}{b_{\max }}\left(1+\frac{\lambda_{i}(\beta, \rho)}{c_{\max }}\right) \leq \widehat{\lambda}_{i} \leq \frac{c_{\max }}{b_{\min }}\left(1+\frac{\lambda_{i}(\beta, \rho)}{c_{\min }}\right)$;

(iii) we can choose $P^{\prime}$ as $P^{\prime}=P$.

Proof. a) We first prove an auxiliary result: let $D$ be a nonnegative symmetric $k \times k$ matrix, and $\Gamma$ be a diagonal matrix with $\Gamma_{i, i}>0$, and $D^{\prime}=\Gamma D \Gamma$. Denote by $\lambda_{i}$ and $\lambda_{i}^{\prime}$ the eigenvalues of $D$ and $D^{\prime}$, increasingly ordered. Letting $\Gamma_{\max }=\sup _{i} \Gamma_{i, i}$ and $\Gamma_{\min }=\inf _{i} \Gamma_{i, i}$, we have

$$
\Gamma_{\min }^{2} \lambda_{i} \leq \lambda_{i}^{\prime} \leq \Gamma_{\max }^{2} \lambda_{i}
$$


To see this, let $\mathcal{E}_{i}$ be the family of all linear subspaces of $\mathbb{R}^{k}$ with dimension $i$. We have $\lambda_{i}=\inf _{E \in \mathcal{E}_{i}} \sup \left(x^{*} D x\right.$ : $x \in E,\|x\|=1$ ), and similarly for $\lambda_{i}^{\prime}$. The map $E \mapsto \Gamma E$ (image of $E$ by $\Gamma$ ) is one-to-one and onto from $\mathcal{E}_{i}$ into itself, hence

$$
\begin{aligned}
\lambda_{i}^{\prime} & =\inf _{E \in \mathcal{E}_{i}} \sup \left((\Gamma x)^{*} D \Gamma x: x \in E,\|x\|=1\right) \\
& =\inf _{E \in \mathcal{E}_{i}} \sup \left(y^{*} D y: y \in E,\left\|\Gamma^{-1} y\right\|=1\right) .
\end{aligned}
$$

Now if $\left\|\Gamma^{-1} y\right\|=1$ we have $\Gamma_{\min } \leq\|y\| \leq \Gamma_{\max }$, and (8.5) follows.

b) Set $D=D(\beta, \rho)$. Denote by $M$ the diagonal matrix with entries $M_{i, i}=\sqrt{c_{i} / b_{i}}$, and $L=M^{-1} \Delta D \Delta M^{-1}$ and $N=I+L$, so that

$$
\widehat{C}=M^{2}+\Delta D \Delta=M N M .
$$

Next, denote by $\lambda_{i}(D)=\lambda_{i}(\beta, \rho), \lambda_{i}(L)$ and $\lambda_{i}(N)=\lambda_{i}(L)+1$ the eigenvalues of $D, L$ and $N$ respectively, increasingly ordered. Since $\left(\Delta M^{-1}\right)_{i, i}=\sqrt{1 / c_{i}}$, applying twice (8.5) yields

$$
\begin{gathered}
\frac{1}{c_{\max }} \lambda_{i}(D) \leq \lambda_{i}(L) \leq \frac{1}{c_{\min }} \lambda_{i}(D), \\
\frac{c_{\min }}{b_{\max }} \lambda_{i}(N) \leq \hat{\lambda}_{i} \leq \frac{c_{\max }}{b_{\min }} \lambda_{i}(N),
\end{gathered}
$$

hence (ii). Next, (i) readily follows from $\widehat{C}^{\prime}=\widehat{C}+a I$. Finally, $\Lambda^{\prime}=\Lambda+a I$ yields for any possible choice of $P$ and $P^{\prime}$ :

and (iii) follows.

$$
P^{\prime} \Lambda P^{\prime *}+a I=\widehat{C}^{\prime}=\widehat{C}+a I=P \Lambda P^{*}+a I,
$$

Finally, in connection with the eigenvalues given in (8.2), we introduce the function $\phi(x, a)=2(1-\cos (x))+a$ on $(0, \infty) \times \mathbb{R}$. We need the following simple properties of integrals and Riemann sums:

First, for any $a>0$ the following integral may be explicitely computed:

$$
I_{2}(a):=\int_{0}^{\pi} \frac{1}{\phi(x, a)^{2}} \mathrm{~d} x=\frac{\pi(2+a)}{a^{3 / 2}(4+a)^{3 / 2}} .
$$

Next, define the Riemann sums, for $k=2,3, \ldots$ :

$$
J_{2}(a, k)=\frac{\pi}{k} \sum_{i=1}^{k-1} \frac{1}{\phi\left(\frac{i \pi}{k}, a\right)^{2}}, \quad J_{2}^{\prime}(a, k)=\frac{\pi}{k} \sum_{i=0}^{k-1} \frac{1}{\phi\left(\frac{i \pi}{k}, a\right)^{2}} .
$$

We then have the following inequalities:

$$
I_{2}(a)-\frac{\pi}{k a^{2}} \leq J_{2}(a, k) \leq I_{2}(a) \leq J_{2}^{\prime}(a, k) \leq I_{2}(a)+\frac{\pi}{k a^{2}} .
$$

\section{REFERENCES}

[1] P. Bickel and Y. Ritov, Inference in hidden Markov models. I. Local asymptotic normality in the stationary case. Bernoulli 2 (1996) 199-228.

[2] P. Bickel and Y. Ritov, Asymptotic normality for the maximum likelihood estimator for general hidden Markov models. Ann. Statist. 26 (1998) 1614-1635.

[3] G. Dohnal, On estimating the diffusion coefficient. J. Appl. Probab. 24 (1987) 105-114.

[4] V. Genon-Catalot and J. Jacod, On the estimation of the diffusion coefficient for multidimensional diffusion processes. Ann. Inst. H. Poincaré Probab. Statist. 29 (1993) 119-153.

[5] V. Genon-Catalot and J. Jacod, Estimation of the diffusion coefficient for diffusion processes: random sampling. Scand. J. Statist. 21 (1994) 193-221.

[6] A. Gloter and J. Jacod, Diffusion with measurement error. II. Optimal estimators (2000). 
[7] E. Gobet, LAMN property for elliptic diffusions (2000).

[8] J. Jacod and A. Shiryaev, Limit Theorems for Stochastic Processes. Springer-Verlag, Berlin (1987).

[9] J.L. Jensen and N. Petersen, Asymptotic normality of the Maximum likelihood estimator in state space models. Ann. Statist. 27 (1999) 514-535.

[10] B. Leroux, Maximum likelihood estimation for hidden Markov models. Stochastic Process. Appl. 40 (1992) 127-143.

[11] L. LeCam and G.L. Yang, Asymptotics in Statistics. Springer-Verlag, Berlin (1990).

[12] M.B. Malyutov and O. Bayborodin, Fitting diffusion and trend in noise via Mercer expansion, in Proc. Yth Int. Conf. on Analytical and Stochastic Modeling Techniques. Hamburg (2000).

[13] T. Ryden, Consistent and asymptotically normal estimators for hidden Markov models. Ann. Statist. 22 (1994) $1884-1895$. 